\title{
Five Years of Tracking Heavy Ion Collisions at RHIC
}

\author{
Achim Franz \\ Brookhaven National Laboratory, Physics Department, Bld 510C, Upton, NY 11733-5000, USA
}

\begin{abstract}
Five years have passed since the first collisions of Au nuclei at the Relativistic Heavy Ion Collider (RHIC) at Brookhaven National Laboratory (BNL) on Long Island. With nucleon-nucleon center-of-mass energies of up to $\sqrt{s_{N N}}=200 \mathrm{GeV}$ RHIC provides the highest energy heavy ion collisions at any existing collider. To study the dynamics of nuclear matter at extreme temperatures and pressures hundreds of produced particles need to be tracked and identified, which provides a sizable challenge to the four experiments. This article tries to summarize these first years of RHIC operation from the detector point of view and give a glimpse at the future of the accelerator and its experiments.
\end{abstract}

Key words: RHIC, heavy ion, collider, tracking $P A C S$ :

\section{Introduction}

The history of RHIC and its experiments goes back to the early 1980's and is summarized e.g. in [1]. After an initial engineering run in 1999 cooldown of the two $3.8 \mathrm{~km}$ long cryostats containing about 1000 super-conducting magnets started in February 2000 and operation with gold beams, delivered by the injection chain consisting of Tandem, Booster, and AGS, started in April. First collisions in all four experiments were observed during June 2000 and a luminosity of $\mathcal{L}=2 \times 10^{25} \mathrm{~cm}^{-2} \mathrm{~s}^{-1}$ (10\% of design) has been achieved.

Since then a total of five runs have produced billions of events, peta-bytes (PB) of data, 0.5 PB for the 2004/2005 PHENIX dataset alone, at various beam energies and collision species of up to twice the design luminosity, see Table 1 , resulting in 122 articles in peer-reviewed journals and 103
$\mathrm{PhD}$ thesis to-date. A comprehensive summary of the physics results from the four experiments covering the first three years is available in [2].

\section{The RHIC complex}

Figure 1 shows the layout of RHIC for Au running [3]. Ions from the Tandem are accelerated in the Booster then collected and bunched in the AGS and injected into the two separate $3.8 \mathrm{~km}$ long RHIC rings. Protons for the polarized proton program are injected from a Linac, middle-left on Fig.1. A complete overview of the RHIC accelerator complex can be found in [4].

Already in the second year of operation RHIC achieved design luminosity, but with the drawback of high backgrounds and short times in store mainly due to intra beam scattering. A better 


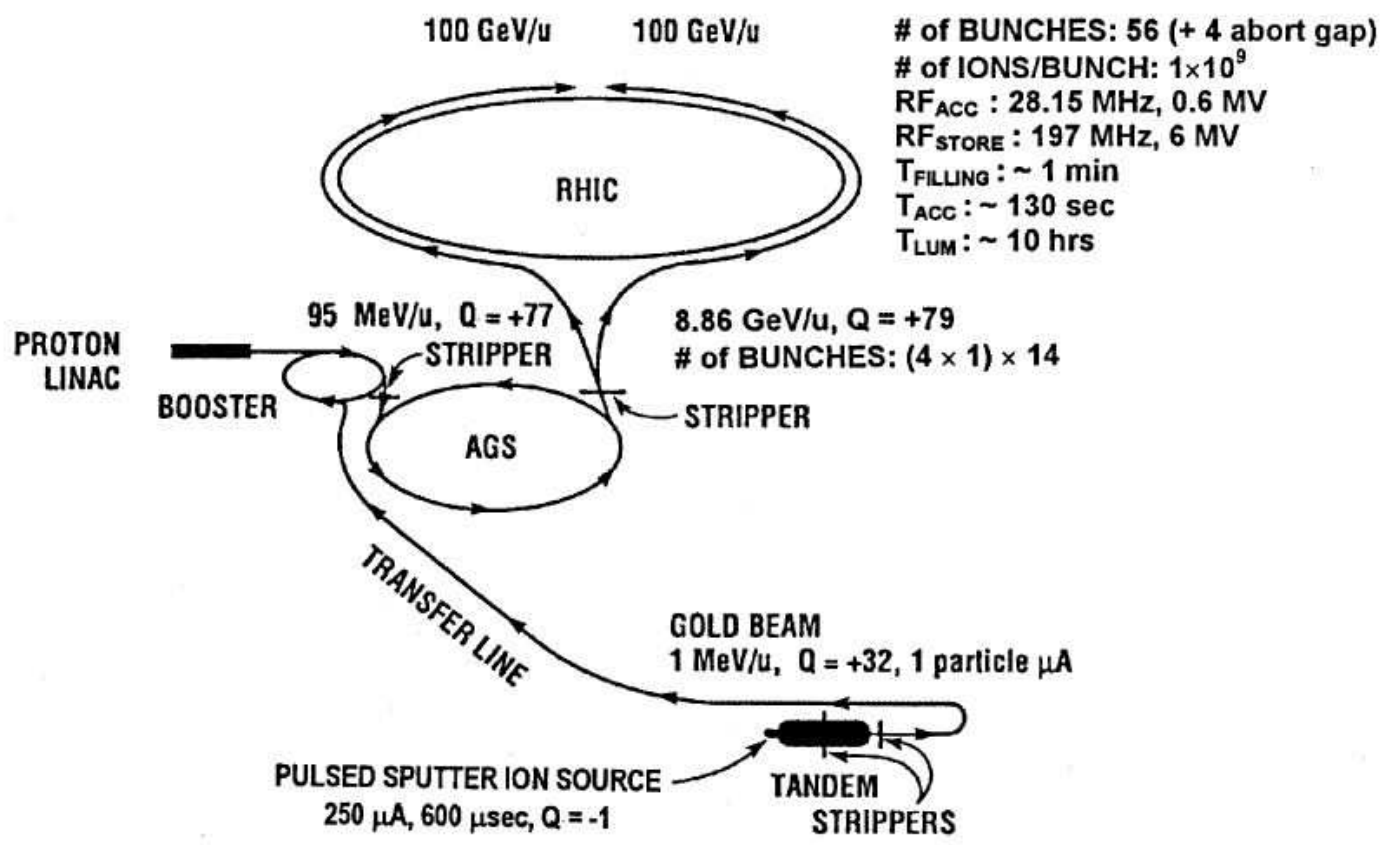

Fig. 1. The RHIC complex for Au collisions [3].

vacuum in the beam pipes, especially the warm sections, was achieved by bake-out and NEG coating. The use of Storage RF and collimators have further improved the beam quality. However the experiments were still suffering from the background levels, specially during the beginning of each store. This made the operation of some wire chambers impossible, e.g. the muon chambers installed in PHENIX suffered from rates 100 times higher than was estimated from collisions and expected beam background. Therefore, extensive shielding had to be installed in the tunnel close to the interaction regions. Measurements close to the beam-pipe at the PHOBOS experiment indicate an average dose of $1 \mathrm{kRad} /$ year [5].

Details about the RHIC performance of the last years can be found in Table 1, the RHIC run summary web page [6], and in the proceedings of recent (European) Particle Accelerator Conferences (PAC, EPAC) $[7,8]$.

\section{The Experiments}

Before data from the RHIC experiments were available, models predicted the charged particle multiplicity at mid-rapidity to be between 600 1400 particles. Consequently the experiments were designed to cope with the highest multiplicities predicted.

Comparing the first results, see Fig. 2, presented at the Quark Matter Conference 2001 with model predictions at that time [9] revealed that the measured multiplicity was at the low end of the spectrum. This not only aided the RHIC experiments but made it feasible for the LHC p-p experiments to participate in the heavy ion program, where up to 8000 charged particles are expected.

Figure 3 shows a set of charged particle multiplicity measurements for three different collision systems, four energies and multiple collision centralities [10]. These RHIC data combined with SPS measurements extrapolate to a LHC Pb-Pb multiplicity of around 1000 - 2000 at mid-rapidity. How the LHC experiment CMS is preparing for the 
Table 1

RHIC operating modes and total integrated luminosity delivered to the experiments [6]

\begin{tabular}{|c|c|c|c|c|}
\hline runs & species & $\begin{array}{c}\text { particle } \\
\text { energy } \\
{[\mathrm{GeV} / \mathrm{n}]}\end{array}$ & $\begin{array}{c}\text { total } \\
\text { delivered } \\
\text { luminosity }\end{array}$ & $\begin{array}{c}\text { average } \\
\text { store } \\
\text { polarization }\end{array}$ \\
\hline \multirow[t]{2}{*}{ Run-1 2000} & $A u^{79+}-A u^{79+}$ & 27.9 & $<0.001 \mu b^{-1}$ & \\
\hline & $A u^{79+}-A u^{79+}$ & 65.2 & $20.0 \mu b^{-1}$ & \\
\hline \multirow[t]{3}{*}{ Run-2 2001/02 } & $A u^{79+}-A u^{79+}$ & 100.0 & $258.0 \mu b^{-1}$ & \\
\hline & $A u^{79+}-A u^{79+}$ & 9.8 & $0.4 \mu b^{-1}$ & \\
\hline & $p^{+}-p^{+}$ & 100.0 & $1.4 p b^{-1}$ & $14 \%$ \\
\hline \multirow[t]{2}{*}{ Run-3 2002/03 } & $d^{+}-A u^{79+}$ & 100.0 & $73.0 n b^{-1}$ & \\
\hline & $p^{+}-p^{+}$ & 100.0 & $5.5 p b^{-1}$ & $34 \%$ \\
\hline \multirow[t]{3}{*}{ Run-4 2003/04 } & $A u^{79+}-A u^{79+}$ & 100.0 & $3740.0 \mu b^{-1}$ & \multirow[b]{3}{*}{$46 \%$} \\
\hline & $A u^{79+}-A u^{79+}$ & 31.2 & $67.0 \mu b^{-1}$ & \\
\hline & $p^{+}-p^{+}$ & 100.0 & $7.1 p b^{-1}$ & \\
\hline \multirow[t]{5}{*}{ Run-5 2004/05 } & $C u^{29+}-C u^{29+}$ & 100.0 & $42.1 n b^{-1}$ & \\
\hline & $C u^{29+}-C u^{29+}$ & 31.2 & $1.5 n b^{-1}$ & \\
\hline & $C u^{29+}-C u^{29+}$ & 11.2 & $0.02 n b^{-1}$ & \\
\hline & $p^{+}-p^{+}$ & 100.0 & $29.5 p b^{-1}$ & $46 \%$ \\
\hline & $p^{+}-p^{+}$ & 204.9 & $0.1 p b^{-1}$ & $30 \%$ \\
\hline
\end{tabular}

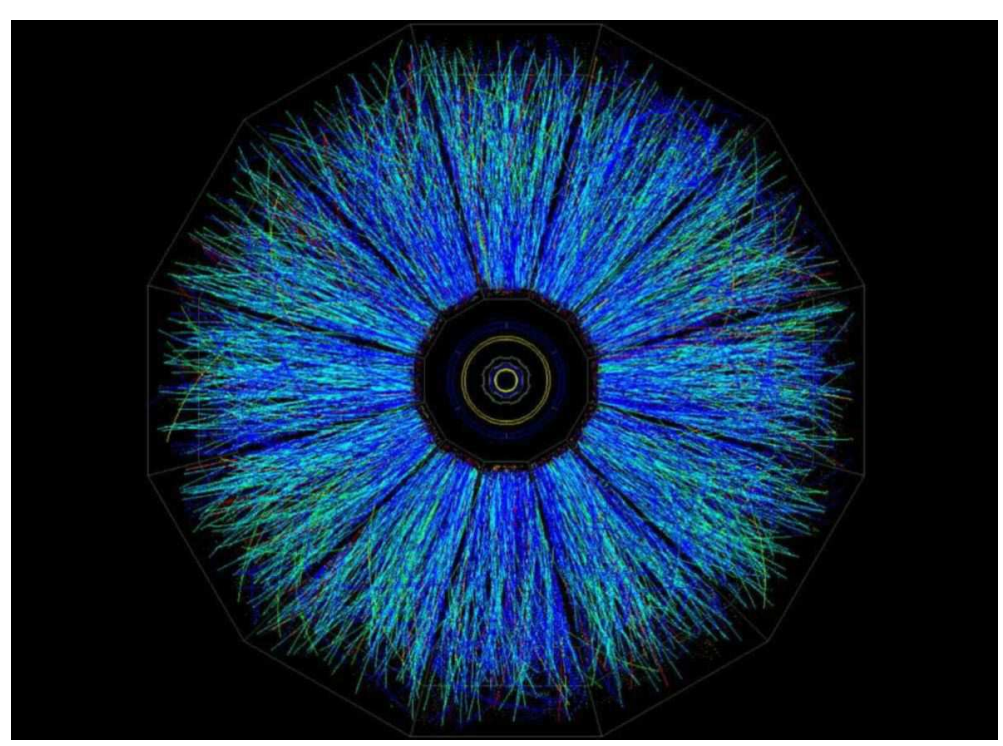

Fig. 2. Beams eye view of a central event in the STAR Time Projection Chamber. This event was drawn by the STAR level-3 online display. 


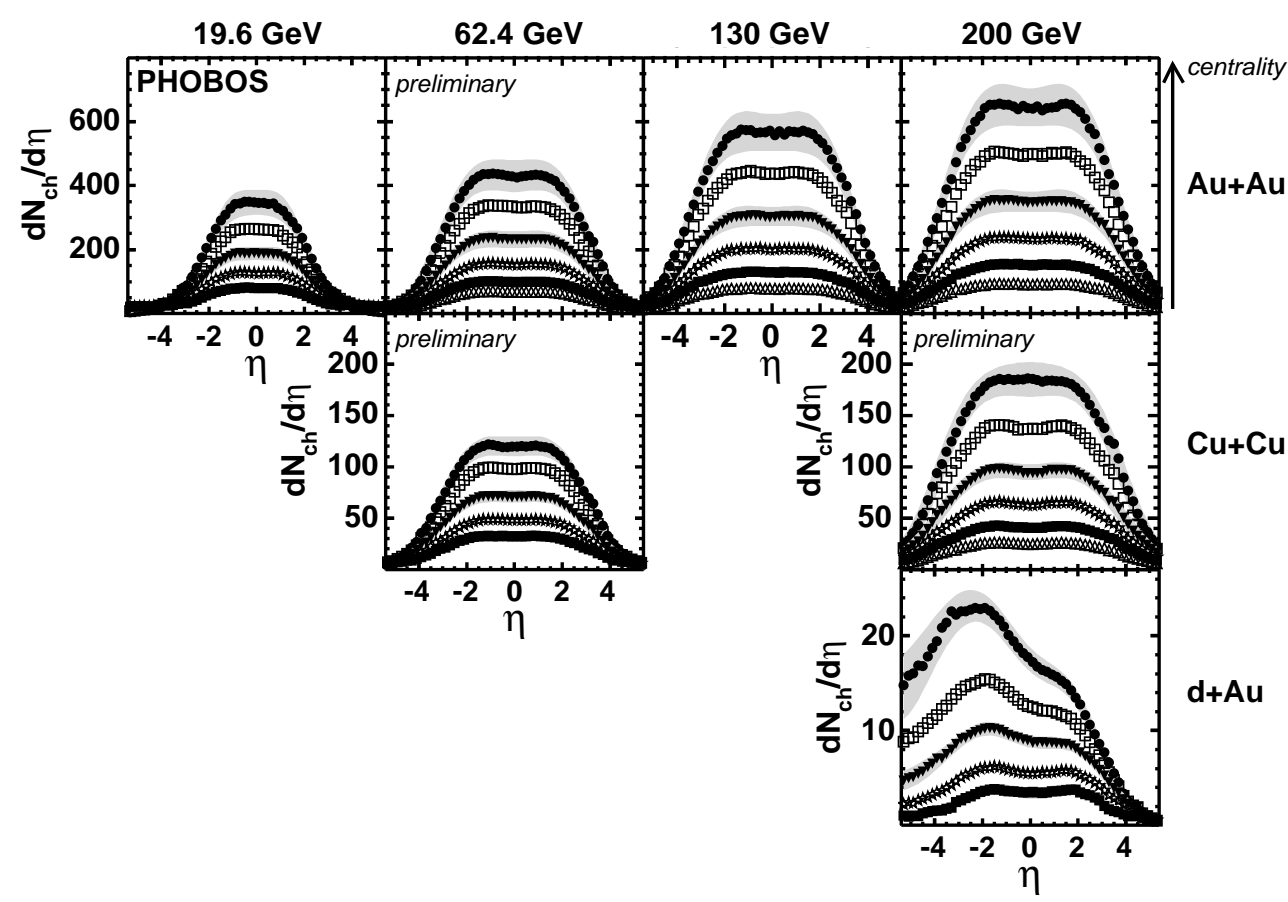

Fig. 3. Charged hadron pseudorapidity distributions for different collisions centralities (see text) for Au-Au collisions at $\sqrt{s_{N N}}=19.6,62.4,130$ and $200 \mathrm{GeV}$ (top row from left to right), $\mathrm{Cu}-\mathrm{Cu}$ at 62.4 and $200 \mathrm{GeV}$ (middle row, preliminary) and d-Au at $200 \mathrm{GeV}$ (bottom row) [10].

heavy ion program can be found in [11].

Heavy ions are extended objects and their collisions is not always head-on, the degree of geometrical overlap, or centrality, is described by the impact parameter, $b$, or perpendicular distance to the closest approach if the ions were undeflected. Zero degree calorimeters (ZDC) $[12,13]$ have been installed in all experiments to provide a collision and vertex location trigger as well as a measure of the collision centrality by the energy deposited from the forward going spectator neutrons. A complete geometrical overlap of the ions, $b \approx 0$, is called a central or large centrality event resulting in e.g. a high charged particle multiplicity and very few or none forward going neutrons. The ZDCs also provide feedback to the accelerator operators for steering and to estimate the delivered luminosity. In addition to the ZDCs all experiments have collision triggers installed, usually quartz Cherenkov or Scintillator detectors, which provide a trigger for the vertex location, in the $\mathrm{cm}$ range, and a start time for drift or time-of-flight (TOF) measurements.

Four experiments are participating in the heavy ion program at RHIC:

Broad Range Hadron Magnetic Spectrometer (BRAHMS) [14].

The collaboration consists of 56 physicists from 12 institutions in 5 countries. The experiment is a small acceptance spectrometer consisting of a set of global detectors for event characterization and two spectrometer arms designed to identify hadrons over a wide range in rapidity and momentum. The two arms are used to cover the mid-rapidity range (MRS $6.5 \mathrm{msr}$ ) where the particle momentum is in the few hundred $\mathrm{MeV} / \mathrm{c}$ range, and a movable $(30<\vartheta<90)$ forward arm (FS $0.8 \mathrm{msr})$ which can identify particles up to $30 \mathrm{GeV} / \mathrm{c}$.

The particle identification (PID) is performed 
with standard spectrometer components like Time-of-Flight (TOF), threshold and ring-imaging Cherenkov detectors and tracking devices like drift (DC) and time-projection (TPC) chambers. The TPCs are of standard design with $21.8 \mathrm{~cm}$ drift $(229 \mathrm{~V} / \mathrm{cm})$ using $\mathrm{Ar} / \mathrm{CO}_{2}$ (90:10) and STAR front-end-electronics (FEE). The DCs have 10 layers with $\mathrm{x}, \mathrm{y}$, and $\pm 18^{\circ}$ wire planes using a $\mathrm{Ar} / \mathrm{C}_{4} \mathrm{H}_{10}(67: 33)$ gas mixture with a $9^{\circ} \mathrm{C}$ alcohol bubbler. Due to the low track density of 0.01 to 0.1 $\mathrm{cm}^{-2}$ a simple 'follow-your-nose' algorithm is used for tracking. This is a simple approach in which a hypothesis or previously determined points are used to predict where the next measured point on the track should be.

The compact size of the detectors are ideal for construction and maintenance. In one of the earlier runs a field wire in the TPC broke, but being $<0.2 m^{3}$ in size they can easily be extracted and repaired in the lab. None of the tracking chambers as shown any aging effects.

Pioneering High Energy Interaction Experiment (PHENIX) [15].

The collaboration consists of 498 physicists from 62 institutions in 13 countries. The experiments was designed to measure mainly electrons, muons, and photons, with a good hadron PID at midrapidity. It has two distinct detector sections. At mid-rapidity two arms with tracking, PID and calorimetry measures hadrons, leptons and photons, whereas in the forwards directions muons are identified and tracked.

The muon tracking system [16] uses three layers of cathode strip chambers inside the muon magnets and 6 layers of Iarocci drift tubes interleaved with several inches of steel. These chambers suffered the most from high beam background but no aging effects were observed. Because they are located inside the magnetic field the strip chambers include an optical alignment system [17].

The mid-rapidity arms [18] feature, apart from PID detectors, three types of wire chambers, a jet-type drift- (DC), 2(3) layers of pad- and 6 layers of time-expansion/transition radiation(TEC/TRD) chambers. Early on HV instabilities required the addition of an alcohol bubbler $\left(0^{\circ} \mathrm{C}\right)$ to the DC gas of $A r / C_{2} H_{6}(50: 50)$. The TEC/TRD
( $\mathrm{Xe} / \mathrm{He} / \mathrm{CH}_{4}$ 45:45:10) shows a slight decrease in gain over the past few years which could be a sign of material induced aging or deposits on the field wires.

In 2005 the multiplicity-vertex detector (MVD) [19], a silicon strip (barrel) and pad (end-cap) detector, was removed from the setup because noise induced fluctuations in the pedestal values made reliable physics measurements impossible. It will be replaced in the coming future by a multi-layer vertex tracking detector [20].

PHOBOS [21]. The collaboration consists of 68 physicists from 8 institutions in 3 countries. The name of the experiment is not an abbreviation, it was chosen from the name of one of the moons of Mars, which was the first name of the experiment.

Its goal was to study particle production down to very low $p_{T}, 10 \mathrm{MeV} / \mathrm{c}$ for detectable and $30 \mathrm{MeV} / \mathrm{c}$ for momentum analyzed, over a large phase space. Four subsystems make up the experiment, see Fig.4 a multiplicity array, a vertex detector, a two arm magnetic spectrometer including TOF, and several trigger detectors which also determine the centrality of the collision. Three of those consists of silicon pad arrays with a total 9 different silicon wafers with varying pad sizes. Details about these can be found in [21] and information about tracking and vertex algorithms used in $[22,23]$. After another very successful run PHOBOS was shutdown in 2005.

Solenoidal Tracker at RHIC (STAR) [24]. The collaboration consists of 616 physicists from 52 institutions in 12 countries, This experiment excels in measuring hadron production over a large solid angle. It features detector systems for high precision tracking, momentum analysis, and particle identification at center-of-mass rapidity. The centerpiece is the large TPC $\left(\mathrm{Ar} / \mathrm{CH}_{4}\right.$ 90:10) with its two $210 \mathrm{~cm}$ drift regions $(|\eta|<1.8)$ for tracking and PID together with a 3 layer silicon vertex tracker (SVT), a single layer silicon strip detector (SSD) and electromagnetic calorimetry around the TPC. Tracking of the around 2000 charged particles per central event in the TPC is performed by a Kalman Filter and Hough transform which points the tracks back to the SVT/SSD. Smaller 


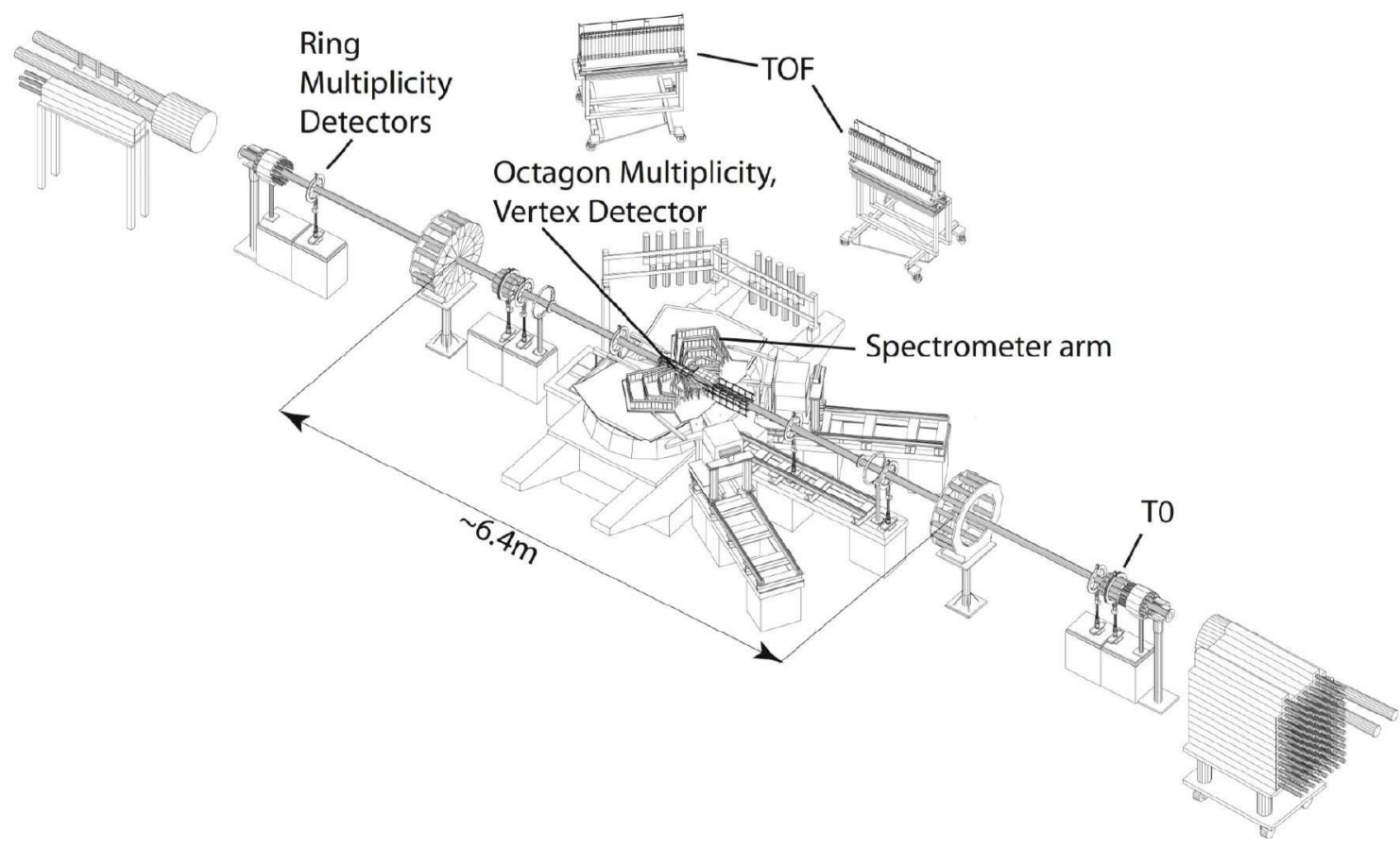

Fig. 4. Layout of the PHOBOS experiment in 2003, the top part of the magnet around the collision point is removed for clarity.

angles, $\theta=2^{\circ}-9^{\circ}$, are covered by two small forward-TPCs $\left(\mathrm{Ar} / \mathrm{CO}_{2} 50: 50,23 \mathrm{~cm}\right.$ drift, 240$1400 \mathrm{~V} / \mathrm{cm}$ ) with radial drift. Not only is the large volume of the TPC very sensitive to beam induced background, but the high collision rate at the large luminosities have introduced space charge problems as described in [25]. Over the years the SVT has suffered problems with a reset to its front-end electronics (FEE) crates which eliminated it from the data-stream for some fraction of events.

All experiments have performed beyond their expectations despite startup problems in the early runs. No beam induced aging has been observed in any of the tracking devices, most problems were related to the FEE. Small improvements and additions were made over the years, but more substantial upgrades are necessary to deal with future machine improvements.

\section{Upgrades}

The quest to understand the new form of matter created in these nucleus collisions demand higher luminosities, which can be achieved by more numerous and smaller bunches, faster data acquisition (DAQ) rates, and new specialized detector systems. Beyond 2008 RHIC will also compete with the heavy ion program at the LHC and needs to exploit its unique spin physics program. Accelerator and detector upgrades are being proposed and built to keep RHIC and its experiments on the leading edge. A complete summary can be found in the reviews, white-papers and decadal plans in [30].

\subsection{RHIC upgrades}

In the near future RHIC will upgrade its aging Tandem Van-de-Graaffs with an Electron Beam Ion Source (EBIS) [26]. This source and linac based pre-injector can produce all ion species up to uranium, including noble gases and polarized $\mathrm{He}^{3}$ and 
switch from one species to another within seconds to provide RHIC and the NASA Space Radiation Laboratory (NSRL) at BNL with a variety of ion species. EBIS can go into operation around 2009.

Currently intra-beam scattering limits the size and intensity of RHIC bunches. To achieve the planned upgrade to $40 \mathrm{x}$ design luminosity of RHIC-II electron cooling is needed. Electron cooling of a $100 \mathrm{GeV} \mathrm{Au} \mathrm{beam} \mathrm{with} \mathrm{about} 10^{9}$ ions per bunch would require a $54 \mathrm{MeV}$ electron beam of 100-200 mA current [27]. Prototype designs are underway for the required $26 \mathrm{~m}$ long, 2-5 T superconduction solenoid magnets, which require $10^{-5}$ field precision.

A high energy, high luminosity polarized electron-ion collider could study the fundamental structure of matter using deep inelastic scattering, RHIC provides already the ion part, so a eRHIC ZDR [28] has been prepared which considers various aspects of the accelerator design. The main design being a $5-10 \mathrm{GeV}$ polarized electron $(10 \mathrm{GeV}$ positron) beam circulating in a storage ring which incepts the ion or polarized proton beam of RHIC in one interaction region. The expected luminosities for $10 \mathrm{GeV}$ e on $250 \mathrm{GeV}$ p are $\mathcal{L}=$ $10^{32}-10^{33} \mathrm{~cm}^{-2} \mathrm{~s}^{-1}$ and $\mathcal{L}=10^{30}-10^{31} \mathrm{~cm}^{-2} \mathrm{~s}^{-1}$ for the $10 \mathrm{GeV}$ e on $100 \mathrm{GeV}$ ion beams. $70 \%$ polarization, transverse and longitudinal, is expected for the lepton and proton beams.

\subsection{Experimental upgrades}

The RHIC baseline program will come to a close in a few years with the completion of PHOBOS (2005) and later BRAHMS. To enhance their detector capabilities over the coming years PHENIX and STAR have developed R\&D programs to cover the following main physics goals [29]:

- Study of quark-gluon matter at high temperatures with AA, pA and pp collisions:

- Jet tomography, including identified hadrons in the $p_{T}$ range $3-10 \mathrm{GeV} / \mathrm{c}$, hadron-jet and $\gamma-j$ et correlations, as well as flavor tagging.

- Thermal radiation and effects of chiral symmetry restoration in the electron-pair continuum, in particular at low masses $\left(<1 \mathrm{GeV} / \mathrm{c}^{2}\right)$.

- Production of mesons with open charm and beauty.

- Quarkonia spectroscopy with $J / \psi, \psi^{\prime}, \chi_{c}$, $\Upsilon(1 s), \Upsilon(2 s), \Upsilon(3 s)$.

- The extended exploration of the spin structure of the nucleon:

- Gluon spin structure $(\Delta G / G)$ with heavy flavour and $\gamma-$ jet correlations.

- Quark spin structure $(\Delta q / q)$ with Wproduction.

- Transversity with jets.

- Exploration of the nucleon structure in nuclei: $\mathrm{A}-, p_{T^{-}}, \mathrm{x}$-dependence of the parton structure of nuclei.

Table 2 puts the upgrade plans into perspective with the physics goals they cover and indicates their time-frame and references.

A Hadron Blind Detector (HBD) will be installed close to the collision point inside the PHENIX central magnet. Its a windowless Cherenkov detector with $C F_{4}$ as the radiator and chamber gas. The readout consists of a triple GEM chamber with a CsI coating on the top layer and pad read-out, see Fig.5. The pad size is slightly smaller than the blob size expected from a passing hadron, resulting in a nearly $100 \%$ probability for a single pad hit. Electrons are expected to produce about 35 photo-electrons which spread over at most 3 pads with the chance of a single-pad hit being negligibly small. The relatively large pad size results also in a low granularity and therefore a low cost detector. In addition, since the photoelectrons produced by a single electron will be distributed between at most three pads, one can expect a primary charge of at least 10 electrons/pad, allowing operation of the detector at a relatively moderate gain of a few times $10^{3}$. Figure 5 illustrates the arrangement of the detector where the emitted electron is reflected by the electric field and amplified by the GEM layers which in return shields the CsI layer from photons produced in the avalanche.

Both PHENIX and STAR plan to update their tracking and offset vertex reconstruction capabilities by new silicon vertex detectors. PHENIX foresees a 4 layer barrel section with pixels at $\mathrm{r}=2.5$ and $5.0 \mathrm{~cm}$ and stripixels [41] at $\mathrm{r}=10$ and $14 \mathrm{~cm}$, read out by the ALICE1LHCb and the FNAL SVX4 chip. In the forward direction 4 umbrella shaped silicon strip detectors will aid vertex reconstruc- 
Table 2

Upgrade plans for PHENIX and STAR and their relevance to the physics goals, X - upgrade critical for success, O - upgrade significantly enhances program

\begin{tabular}{|c|c|c|c|c|c|c|c|c|}
\hline \multirow[t]{2}{*}{ Upgrades } & \multirow{2}{*}{$\begin{array}{l}\text { time } \\
\text { frame }\end{array}$} & \multicolumn{4}{|c|}{ High T QCD... QGP } & \multicolumn{2}{|c|}{ Spin } & \multirow[t]{2}{*}{ Low-x } \\
\hline & & \multicolumn{4}{|c|}{$e^{+} e^{-}$flavor tomography quarkonia } & \multicolumn{2}{|c|}{$\mathrm{W} \Delta G / G$} & \\
\hline \multicolumn{9}{|l|}{ PHENIX [32] } \\
\hline Hadron blind detector [33] & $2006 / 07$ & $\mathrm{X}$ & & & & & & \\
\hline Vertex Tracker $[20,34]$ & $2007 / 08$ & $\mathrm{X}$ & $\mathrm{X}$ & $\mathrm{O}$ & $\mathrm{O}$ & & $\mathrm{X}$ & $\mathrm{O}$ \\
\hline Muon Trigger [35] & 2008 & & & & $\mathrm{O}$ & $\mathrm{X}$ & & \\
\hline Forward cal. [35] & 2009 & & & $\mathrm{O}$ & $\mathrm{O}$ & $\mathrm{O}$ & & $\mathrm{X}$ \\
\hline \multicolumn{9}{|l|}{ STAR [36] } \\
\hline Time of Flight [37] & $2006 / 07$ & & $\mathrm{O}$ & $\mathrm{X}$ & $\mathrm{O}$ & & & \\
\hline heavy flavor tracker $[39,38]$ & 2008 & & $\mathrm{X}$ & & $\mathrm{X}$ & & & \\
\hline Forward Tracker [36] & 2010 & & $\mathrm{O}$ & & & $\mathrm{X}$ & $\mathrm{O}$ & \\
\hline Forward Cal [36] & $2006 / 07$ & & & & & & $\mathrm{O}$ & $\mathrm{X}$ \\
\hline DAQ [36] & $2006 / 09$ & & $\mathrm{O}$ & $\mathrm{X}$ & $\mathrm{X}$ & $\mathrm{O}$ & $\mathrm{O}$ & $\mathrm{O}$ \\
\hline RHIC Luminosity [40] & $2009-$ & $\mathrm{O}$ & $\mathrm{O}$ & $\mathrm{X}$ & $\mathrm{X}$ & $\mathrm{O}$ & $\mathrm{O}$ & $\mathrm{O}$ \\
\hline
\end{tabular}

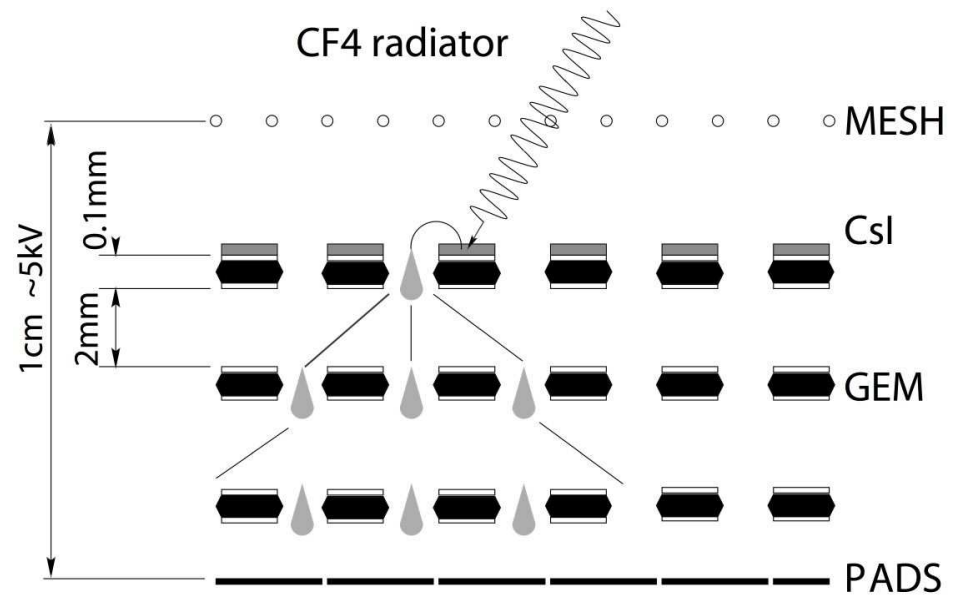

Fig. 5. Proposed triple GEM readout for the PHENIX Hadron Blind Detector

tion and tracking for the muon arms.

STAR has a two fold approach. Two layers of $30 \times 30 \mu m$ CMOS pixels at $\mathrm{r}=1.5$ and $5.0 \mathrm{~cm}$ will reconstruct offset vertices from heavy flavor decays. In a second step a 4 layer silicon detector combined with forward GEM tracking chambers will further enhance the track reconstruction for small $\mathrm{x}$.

To enhance their PID both experiments will install multi step resistive plate chambers (MRPC) as TOF detectors. STAR has several years experi- ence with a small prototype and will complete the full barrel section in a few years. PHENIX will install several modules of the same design in one of the central tracking arms. Single layer RPCs will also be used as trigger chambers for the PHENIX muon arms.

In the very forward direction both experiments will install calorimeters for photon and $\pi^{0}$ detection. PHENIX plans to replace their Brass nosecones with a Nose-Cone-Calorimeter (NCC) made 
from Tungsten plates interleaved with Si pad detectors. STAR will install an array of $\mathrm{Pb}$-glass with photomultiplier readout inside the RHIC tunnel.

To cope with the high rates of the increased luminosity STAR will replace their TPC readout cards with the ALICE design over the next few years

\section{3. $R 2 D$}

To take full advantage of the luminosity available with RHIC II and to undertake detailed measurements of jets, heavy flavors and electromagnetic probes over a large phase space $(-3 \leq \eta \leq 3, \Delta \phi=2 \pi)$, a comprehensive new detector, see Fig.6, has been proposed [42]. Utilizing a high magnetic field $(1.5 \mathrm{~T})$ excellent momentum resolution is foreseen up to $p_{T}=40 \mathrm{GeV} / \mathrm{c}$ and the combination of calorimetry, $\mathrm{dE} / \mathrm{dx}, \mathrm{TOF}$, Aerogel Threshold Cherenkov and RICH counters will provide particle identification and lepton $(\mathrm{e} / \mathrm{h}$, $\pi / \mathrm{h}$ ) separation from 1 up to $20 \mathrm{GeV} / \mathrm{c}$ in $p_{T}$. Even though this new detector would be superior to PHENIX and STAR separately, it does require a major investment. The physics reach beyond what can be achieved with the moderate upgrades of PHENIX and STAR would be the measure to justify its construction.

\section{Summary and Acknowledgment}

The first 5 years of RHIC operations have produced a wealth of data giving new insights into the complex world of high energy nuclear collisions and first glimpse at the role the gluon plays in the proton spin. More than a hundred publications and $\mathrm{PhD}$ thesis and two comprehensive summaries of the project itself and the physics of the first three years have been published. The accelerator has performed better than can be expected from a brand new machine and all the experiments have provided the measurements they were designed for. $\mathrm{R} \& \mathrm{D}$ is ongoing at the experiments and accelerator to further push the luminosity and physics capability of the RHIC complex. In the more distant future eRHIC will open new physic domains to be studied.
The author would like to thank all funding agencies, the staff of all experiments, and the collider group for their professional support to make RHIC such a great success.

\section{References}

[1] G. Baum, Nucl.Phys. A698(2002) xxiii-xxxii

[2] 'First Three Years of RHIC Operation, Physics perspectives from the experiments', Nucl. Phys A 757 (2005), 1-283

[3] H. Hahn et al., NIM A499 (2003) 245-263

[4] M. Harrison, T. Ludlum and S. Ozaki, NIM A499 (2003) 235-880

[5] P. Steinberg, R. Pak, PHOBOS experiment, private communication.

[6] 'run overview of the relativistic heavy ion collider', http://www.agsrhichome.bnl.gov/RHIC/Runs/ assembled by W.Fischer, BNL.

[7] 9th European Particle Accelerator Conference, EPAC04, July 5-9, 2004, Lucerne, Switzerland, http://www.epac04.ch/

[8] 21st Particle Accelerator Conference, PAC05, May 16-20, 2005, Knoxville, TN, USA http://www.sns.gov/pac05/

[9] K.J. Eskola, Nucl.Phys. A698(2002) 78c-87c

[10] 'New Results from the PHOBOS Experiment', G. Roland, QM05 proceedings, arXiv:nucl-ex/0510042 Oct 2005

[11] C. Roland, CMS Heavy Ion Program, these proceedings

[12] A.J. Baltz, C. Chasman, S.N. White, NIM A417 (1989) $1-8$

[13] C. Adler et al., Nucl.Instrum.Meth. A470 (2001) 488499

[14] M. Harrison, T.Ludlum and S.Ozaki, NIM A499 (2003) 437-468, http://www4.rcf.bnl.gov/brahms/WWW/

[15] M. Harrison, T.Ludlum and S.Ozaki, NIM A499 (2003), 469-602, http://www.phenix.bnl.gov/

[16] H. Akikaawa et al., NIM A499 (2003) 537-548

[17] J. Murata et al., NIM A500 (2003) 309-317

[18] K. Adcox et al., NIM A499 (2003) 489-507

[19] M. Allen et al., NIM A499 (2003) 549-559

[20] J. Heuser, NIM A511 (2003) 210-214 


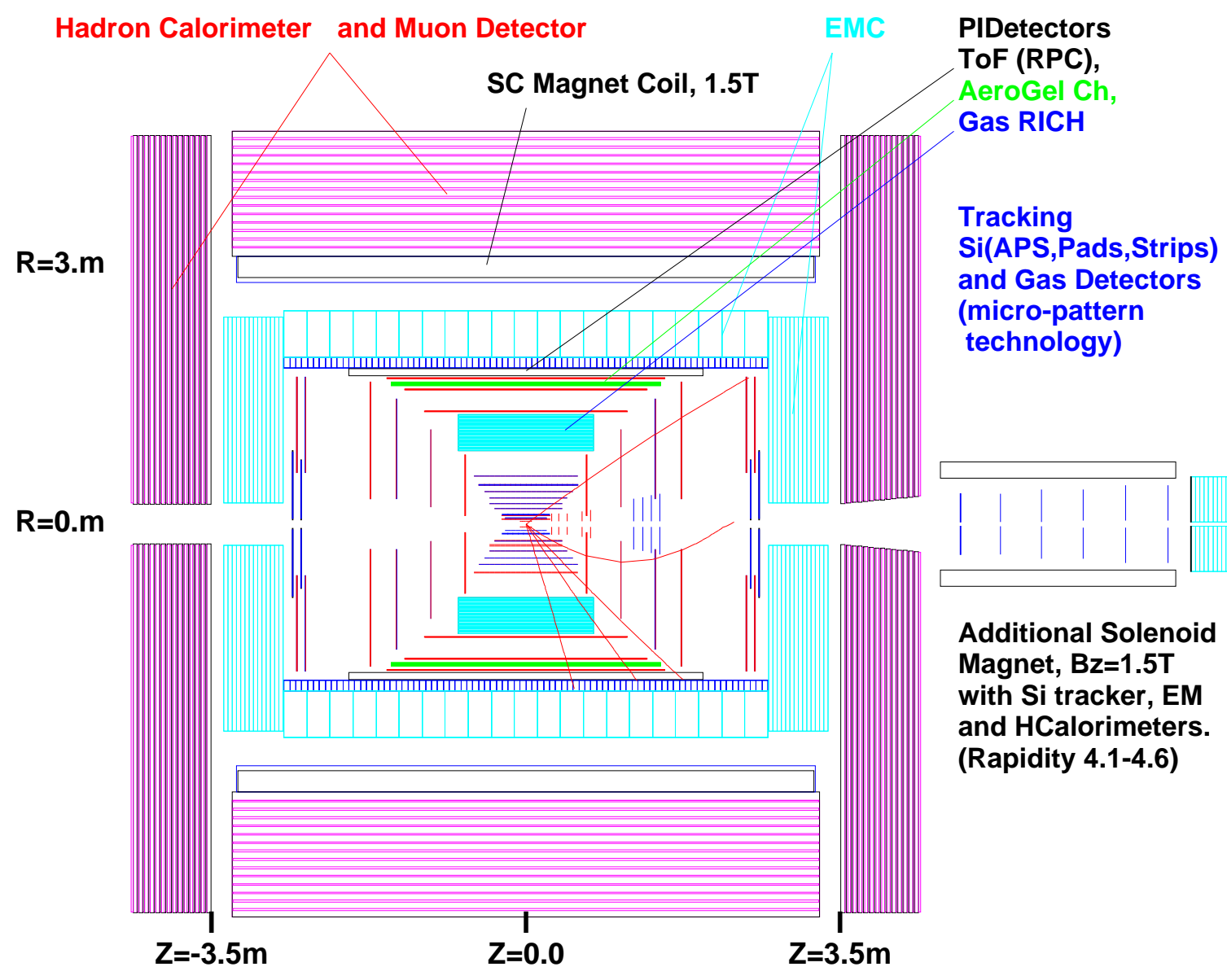

Fig. 6. Diagram of a possible, comprehensive new detector at RHIC II using the SLD magnet [42].

[21] M. Harrison, T.Ludlum and S.Ozaki, NIM A499 (2003), 603-623, http://www.phobos.bnl.gov/

[22] 'Vertex reconstruction algorithms in the PHOBS experiment at RHIC', K. Wozniak these proceedings

[23] 'String banana template method for tracking in highmultiplicity environments with significant multiple scattering', P. Kulinich these proceedings

[24] M. Harrison, T.Ludlum and S.Ozaki, NIM A499 (2003), 624-813, http://www.star.bnl.gov/

[25] 'Correcting for distortions due to Ionization in the STAR TPC', G. vanBuren these proceedings
[26] Progress on test EBIS and the Design of an EBISbased RHIC Preinjector, J.G.Alessi et al., in [8]

[27] Electron Cooling Dynamics for RHIC, A. V. Fedotov et al., AIP Conf. Proc. 773, 415 (2005)

[28] eRHIC Zeroth - Order Design Report, BNL C-A/AP Note 142, 2004, www.agsrhichome.bnl.gov/eRHIC/eRHIC_ZDR.htm eRHIC, A Future Electron-Ion Collider at BNL, V.Ptitsyn et al., MOPLT170 in [7]

[29] A. Drees, J. Phys. G:Nucl. Part.Phys. 30 (2004) S1109S1112

[30] High Energy and Nuclear Physics (HENP) 
Program Advisory Committee web page http://www.bnl.gov/henp/

[31] NSAC Subcommittee on Relativistic Heavy Ions, June 2-6, 2004, BNL, Agenda web page http://nsac2004.bnl.gov/Subc_PublicAgenda_f.htm

[32] 'PHENIX - Future Program', A. Drees in [31]

[33] 'A Hadron Blind Detector for the PHENIX Experiment', I. Ravinovich et al., QM05 proceedings, arXiv:nucl-ex/0510024 Oct 2005

[34] 'Silicon Vertex Tracker', C. Ogilvie in [31]

[35] 'Nose Cone Calorimeter, W boson trigger', K. Barish in $[31]$

[36] 'STAR - Future Program', T. Hallman in [31]

[37] 'PHENIX - TOF Barrel Upgrade', R. Majka in [31]

[38] 'Silicon MicroVertex Detector', H. Wieman in [31]

[39] 'A Heavy Flavor Tracker for STAR', K. Schweda, QM05 proceedings, arXiv:nucl-ex/0510003 Oct 2005

[40] 'RHIC Performance and Plans towards higher Luminosity and higer Polarization', T. Sagotata in [7]

[41] Z. Li et al., NIM A535 (2004) 404-409

[42] J.W. Harris et al., nucl-ex/0407021 P.Steinberg et al., nucl-ex/0503002 